\title{
How to Design Experiments in Animal Behaviour*
}

\section{How Do Wasps Decide Who Would Be the Queen? Part 1}

\section{Raghavendra Gadagkar}

In this, and the next few articles, we will continue to explore the social biology of the primitively eusocial wasp Ropalidia marginata through simple experiments. Since each wasp colony has a single fertile queen and several sterile workers, and since all or most wasps are capable of taking on both roles, the wasps have to decide who will be the queen and who will be the worker/s. Such a decision has to be made both when new colonies are being initiated as well as when an old queen in a mature colony has to be replaced by a new one. Here, I will describe a simple laboratory experiment that reveals that in the context of new nest initiation, wasps decide who will be the queen by fighting - the winner becomes the queen and the loser/s become the worker/s. The same experiment, in addition to revealing the proximate mechanism of the division of reproductive and non-reproductive labour, also throws light on the advantages of such division of labour.

\section{The Paper Wasp Ropalidia marginata}

I have already introduced the Indian paper wasp Ropalidia marginata in the previous article [1]. We need to know a few more facts about this fascinating species, for the purpose of this article (Figure 1). New nests are started either by a lone female (single foundress nests) or by a small group (multiple foundress nests). In single foundress nests, the lone female lays eggs and also performs all the tasks connected with building the nest and caring for the brood. In multiple foundress nests, one of the foundresses becomes the queen and lays eggs while the remaining

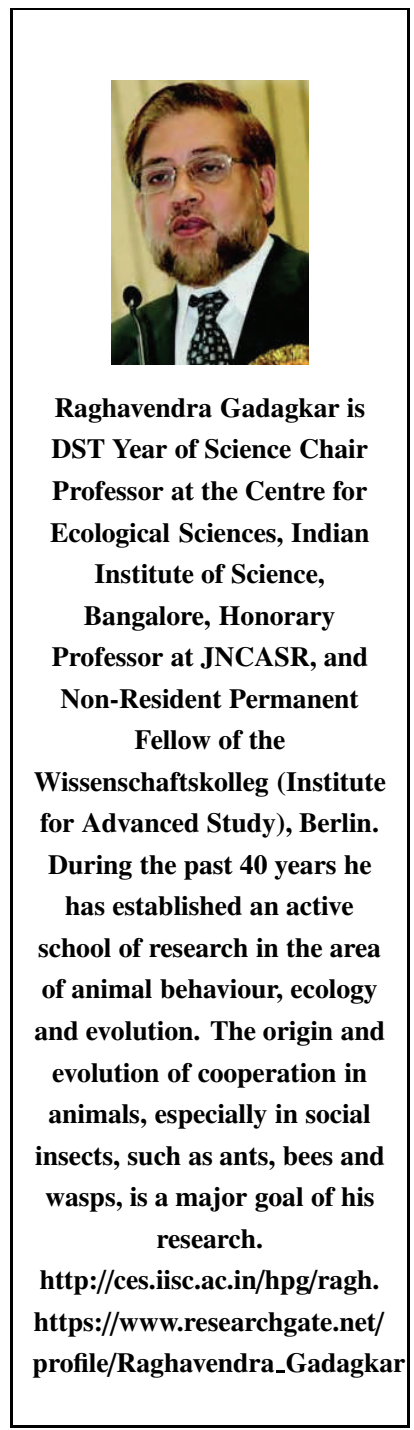


Figure 1. A mature nest of the paper wasp Ropalidia marginata (left), where some but not all of the wasps are marked for individual identification, and newly initiated nests with one, two and three foundresses (right). [Photo credits. Mature nest: Souvik Mandal; Newly initiated nests: Thresiamma Varghese.]

\section{Keywords}

Queen, worker, intranidal and extranidal workers, imago, dominance-subordinate behaviour.

Such queen turnover happens often enough,

but at some random, unpredictable times, and

we are yet to make a systematic study of this most interesting phenomenon-we know rather little about when,

how and why a new individual, let alone which individual, replaces the old queen and becomes the new queen.

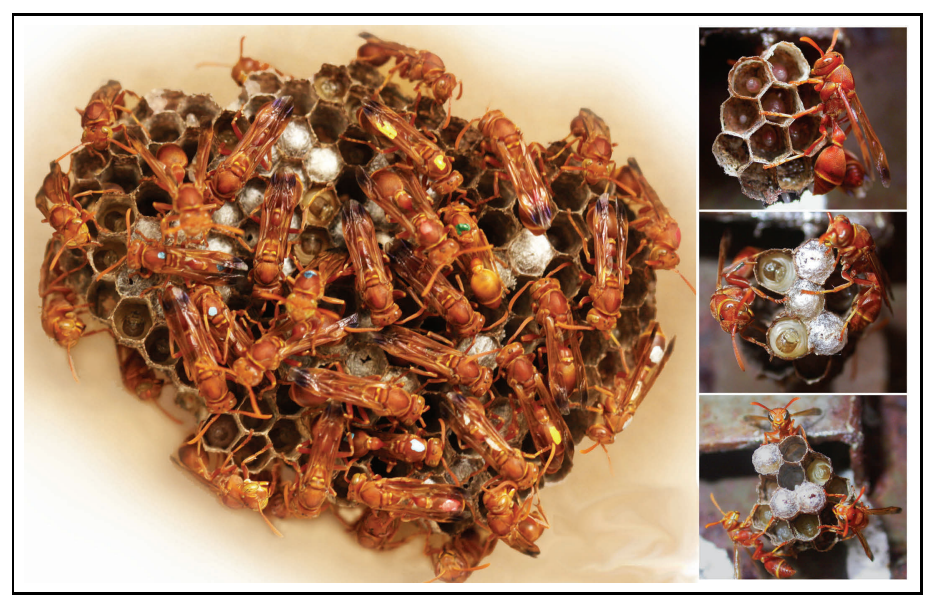

become workers and perform nest building and brood care. Eggs develop into adults, going through the larval and pupal stages, in about 62 days. Eclosing males stay on their parental nests for about a week, after which they leave and lead a nomadic life, mating with females from other colonies, out on foraging trips. Female wasps, however, have at least four different options (Figure 2). Option 1 would be to leave their natal nests and start their own new single foundress nests. Option 2 would be to leave their natal nests but to join other female wasps (usually from the same nest but sometimes from other nests as well) to start new multiple foundress nests. Option 3 would be to stay back in the parental nest and spend their whole lives as non-reproducing workers, helping to rear the queen's brood. Option 4 would be to stay back and work for a while, but then to seek an opportune moment to take over the colony of their birth as its next queen. This may become possible because old queens may age and die or may become too weak and susceptible to challenges by a prospective new queen. Such queen turnover happens often enough, but at some random, unpredictable times, and we are yet to make a systematic study of this most interesting phenomenon-we know rather little about when, how and why a new individual, let alone which individual, replaces the old queen and becomes the new queen. The founding of new single or multiple foundress nests as well as queen turnovers in mature colonies may happen anytime of the year and nests may be abandoned at anytime of the year, 


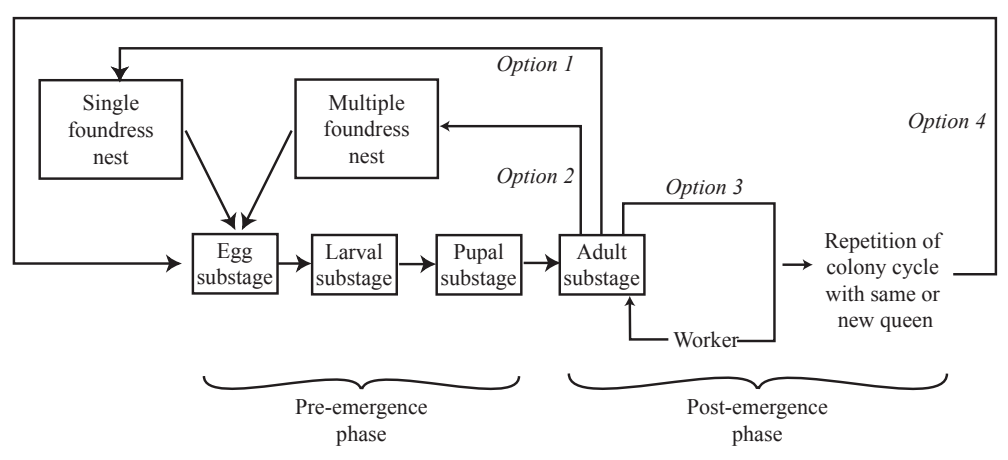

so that we say that $R$. marginata has an almost aseasonal, perennial, indeterminate nesting or colony cycle; 'almost aseasonal', because colonies, both small and large, are more abundant in the summer, though not entirely absent at other times of the year [2].

\section{Caste Differentiation}

One of the reasons we classify $R$. marginata as a primitively eusocial species is because queens and workers are not morphologically differentiated. Mature colonies consist of a single, fertile queen and several non-reproducing workers. There is a clear reproductive division of labour such that queens lay eggs and do little else while workers perform all the other tasks required for the functioning of the colony-cleaning, building, feeding the larvae, guarding, and foraging for food and building material. Whether an individual becomes a queen or a worker is not already fixed at eclosion as in the case of highly eusocial species such as honey bees and ants, but is rather flexible, allowing individuals to switch roles in the adulthood, as suitable opportunities become available or are lost. Such switching is biased-workers frequently switch to queen roles but the reverse seldom happens, although it is not impossible. Workers switch to queen roles under two circumstances, in option 4 described above, when they may replace their previous queens and take over the queen role in the same colony, or in option 2, when they may leave their present colonies and start new ones in which they function as queens. There is a little complication here. Single foundresses are alone in their new
Figure 2. The perennial, indeterminate nesting cycle of $R$. marginata. For schematic convenience, the egg, larval, and pupal stages are shown as being distinct. In reality, there is considerable overlap between them, especially when several colony cycles are repeated on the same nest. Similarly, change of queens can take place at any time in the colony cycle. Note also that new colonies may be initiated at any time of the year and may also be abandoned at any time of the year and at any stage in the colony cycle. Female wasps have at least four different options. (Redrawn with permission from $\mathrm{R}$ Gadagkar. The evolution of eusociality, including a review of the social status of Ropalidia marginata. In: Natural History and Evolution of PaperWasps, (Eds.) S Turillazzi and M J West-Eberhard, Oxford University Press, Oxford, pp.248-271, 1996.) 
colonies and, therefore, have to combine the dual roles of queens and workers, but we will leave that out of consideration for the present [2].

A defining feature of social insect colonies is division of labour, a feature that is thought to be at the root of their tremendous ecological and evolutionary success.
A defining feature of social insect colonies is division of labour, a feature that is thought to be at the root of their tremendous ecological and evolutionary success. Division of labour can be of two kinds-reproductive division of labour, resulting in queens and workers and non-reproductive division of labour resulting in sub-groups of workers specializing in specific tasks such as nursing, foraging or guarding. Groups of individuals specializing in specific tasks are referred to as 'castes'. Thus, we have the queen caste, the worker caste, the forager caste, the soldier caste, etc. A more basic division of non-reproductive labour among the workers is often between those who work at home (intranidal workers) and those who work outdoors (extranidal workers). Soldiers or intranidal workers are sometimes referred to as sub-castes, indicating that this represents a secondary division of labour after the primary division of labour between the queens and workers. The divergence of identical individuals into castes or sub-castes is labelled as caste determination or caste differentiation. How and why does caste differentiation happen in the first place? Recall the distinction between the proximate and ultimate answers to the same question, we encountered in the previous article of this series $[1,3]$. What are the behavioural or physiological mechanisms which bring about the divergence of identical individuals into queens and workers or into intranidal and extranidal workers? Alternatively, what are the evolutionary advantages, or fitness consequences of differentiation into different castes? In this article, we will see that a single, simple experiment can help us answer both these questions. But first, I will focus on the proximate question because that helps answer the question raised in the title of this article, namely, 'Who would be the queen?'. In the end, I will show, as a bonus, that the same experiment also helps answer the ultimate question regarding the advantage of caste differentiation.

The proximate mechanism of caste differentiation depends on 
whether we are focussing on a primitively eusocial species or a highly eusocial species. In highly eusocial species, as we saw above, eclosing adults are already differentiated into queens and workers and cannot reverse their roles anymore. Thus, caste differentiation is said to be 'pre-imaginal'. The adult insect in such holometabolous insects (i.e., those that go through egg, larval and pupal stages before eclosing as adults) is called an 'imago'. Thus, pre-imaginal caste differentiation takes place in the early larval stages and is, therefore, a physiological and developmental phenomenon. We know that differential nutrition leads to differential hormone synthesis and differential development of the female reproductive system. But in the primitively eusocial species, adults are born nearly or entirely totipotent. Here, caste differentiation is post-imaginal, happening in the adult stage - it is, therefore, a behavioural and social phenomenon. Since $R$. marginata is a primitively eusocial species, we are concerned here with the social and behavioural mechanism of caste differentiation. So how do $R$. marginata wasps decide who would be a queen and who would be a worker? This decision has to be made in two contexts-one, when a group of females initiate a new nest and two, when one of the workers has to replace a dead or weak queen in a mature colony. In this article, we will direct our attention to the first context, namely new nest foundation.

\section{The Experiment}

Our goal here is to understand the proximate mechanism of caste differentiation in $R$. marginata, in the context of new nest foundation. In other words, how do the small group of wasps that found a new nest decide which one of them would function as the queen and which one/s would function as non-reproducing workers? This is not a decision that the wasps might be expected to take lightly as queens and workers would be expected to have very different evolutionary fitness. We already know that because $R$. marginata is a primitively eusocial species, caste differentiation is expected to be post-imaginal and mediated through behaviour and social interactions. What we, therefore, need to do is
The proximate mechanism of caste differentiation depends on whether we are focussing on a primitively eusocial species or a highly eusocial species. In highly eusocial species, eclosing adults are already differentiated into queens and workers and cannot reverse their roles anymore.

R. marginata is a primitively eusocial species, caste differentiation is expected to be post-imaginal and mediated through behaviour and social interactions. 
Figure 3. Souvik Mandal (left), Anindita Brahma (center) with the author (right). [Photo: Thresiamma Varghese]

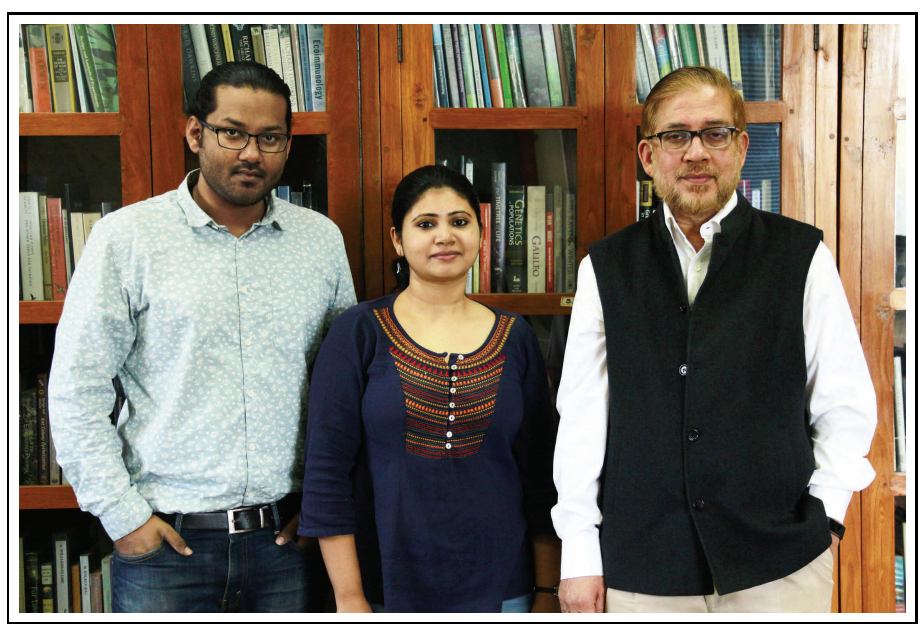

to observe the behaviour of the wasps during the early stages of new nest foundation. This, however, is not as easy as it sounds. In nature, newly initiated nests are hard to find and when we do find them, it is possible that the crucial behavioural interactions that decide who would be the queen has already taken place. It is probably impossible to observe them before they ever interact. And what if they have already interacted and made their decisions before showing up at the new nest? This is precisely the kind of situation where a simplified, artificial and controlled experiment is called for. And that is exactly what my former student Anindita Brahma performed, with help from another former student Souvik Mandal (Figure 3). They collected naturally occurring nests with large numbers of pupae and brought them to the laboratory, leaving the adult wasps behind. Here they removed all the eggs and larvae and waited for the pupae to eclose into adult wasps. Soon after eclosion, adult wasps were isolated into individual holding boxes and prevented from interacting with each other. Then they assigned the wasps to three treatmentssingletons, pairs and triplets. Thus one, two or three randomly chosen wasps were introduced into transparent acrylic boxes and provided with food (Corcyra cephalonica larvae), honey, water and some building material (a piece of soft wood). We know that under these conditions, at least some wasps, whether single or in groups, will initiate a nest, lay eggs and rear them successfully 


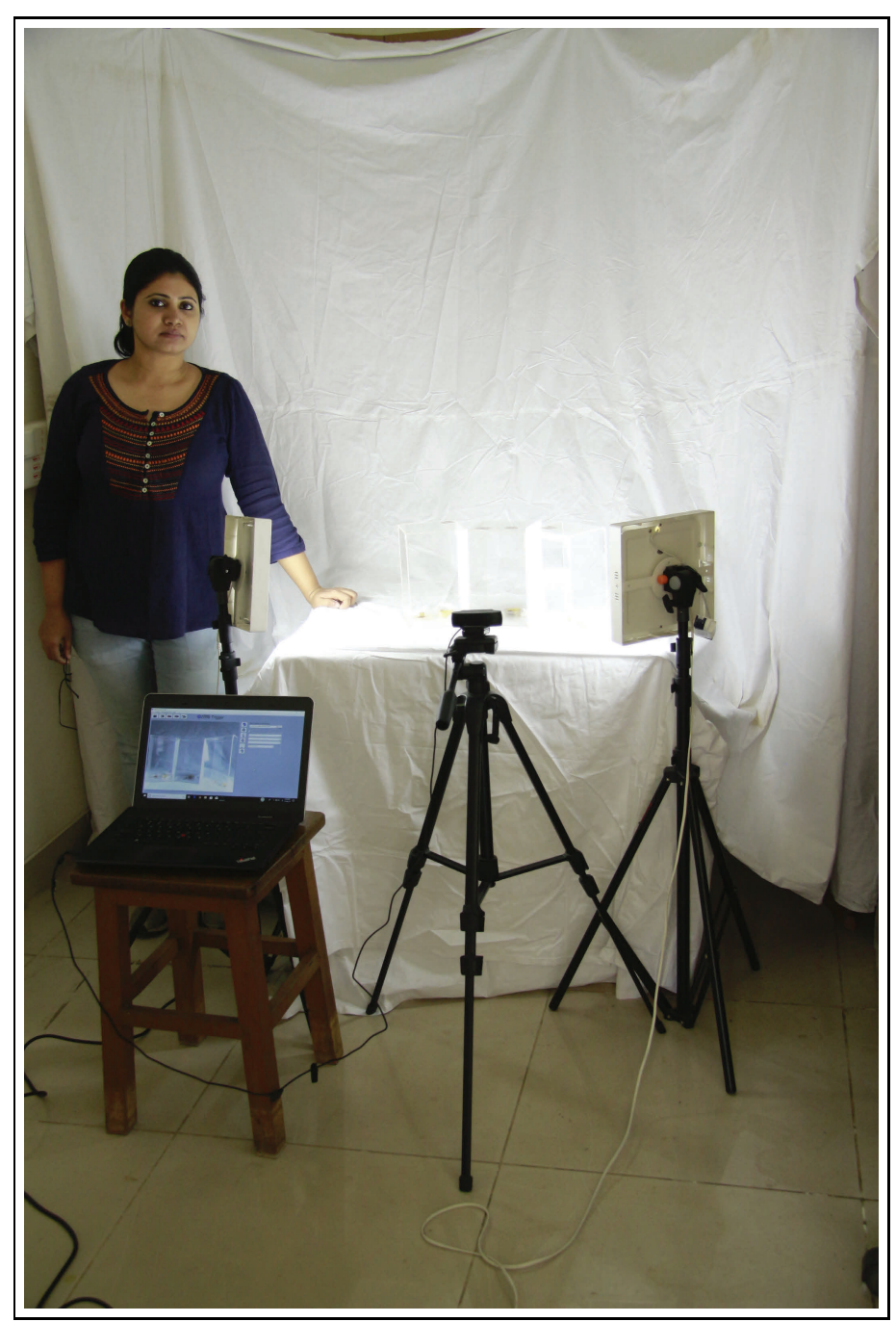

to adulthood. In other words, these conditions simulate new nest foundation in nature. This is quite remarkable because all these wasps are still virgin. Fortunately for us, R. marginata females, although they can mate, appear not to require mating to develop their ovaries and lay viable, haploid, male-destined eggs.

\section{Nest Foundation in the Lab}

Anindita and Souvik set up 77 boxes with one wasp each (singletons), 34 boxes with two wasps each (pairs), and 30 boxes with
Figure 4. Anindita Brahma, with the observation and recording set-up. Acrylic boxes containing 1,2 and 3 wasp nests are faintly visible behind the camera stand. [Photo credit: Souvik Mandal] 
We are not sure why some wasps do not initiate nests under the same conditions under which others do. So, we have previously postulated that not all wasps are born totipotent and that there is a certain amount of pre-imaginal caste bias so that some wasps cannot lay eggs,

but the remaining can become either queens or workers. three wasps each (triplets). They regularly monitored each box for the presence of a nest and, after a nest was built, they kept records of the contents of the nest. They also recorded the behaviour of the wasps in each box, using a video camera, for 10 $\mathrm{h}$ before nest initiation and for $30 \mathrm{~h}$ after nest initiation (Figure 4). At the end of the experiment, i.e., after the nest produced at least one adult offspring or one of the wasps died, they collected all the wasps and dissected them to measure their ovaries. Nests were not initiated in all boxes, but 39 singletons, 23 pairs and 20 triplets, initiated nests and laid eggs. This is consistent with our many earlier experiments in which all isolated wasps do not initiate nests; some die without doing so. Moreover, in the present experiment, we discarded pairs and triplets if even one of the wasps died. We are not sure why some wasps do not initiate nests under the same conditions under which others do. The numbers that don't initiate nests are quite substantial. So, we have previously postulated that not all wasps are born totipotent and that there is a certain amount of pre-imaginal caste bias [4] so that some wasps cannot lay eggs, but the remaining can become either queens or workers. It would be nice to understand why some of them failed to lay eggs, but I have not yet been able to come up with a suitable experiment to do so. So, for the time being, we proceed with our experiments, ignoring the dead wasps and working with the living wasps who appear to be totipotent.

\section{Emergence of Cooperation and Division of Labour}

A study of these 82 boxes (singletons, pairs and triplets put together) with successful nest initiation reveals several remarkable features [5].

\section{Cooperation}

In all the 23 boxes with pairs and 20 boxes with triplets, nest building and brood care was cooperative-both the wasps (in pairs), and all the three wasps (in the triplets), invariably participated in these activities. It was never the case that one of 


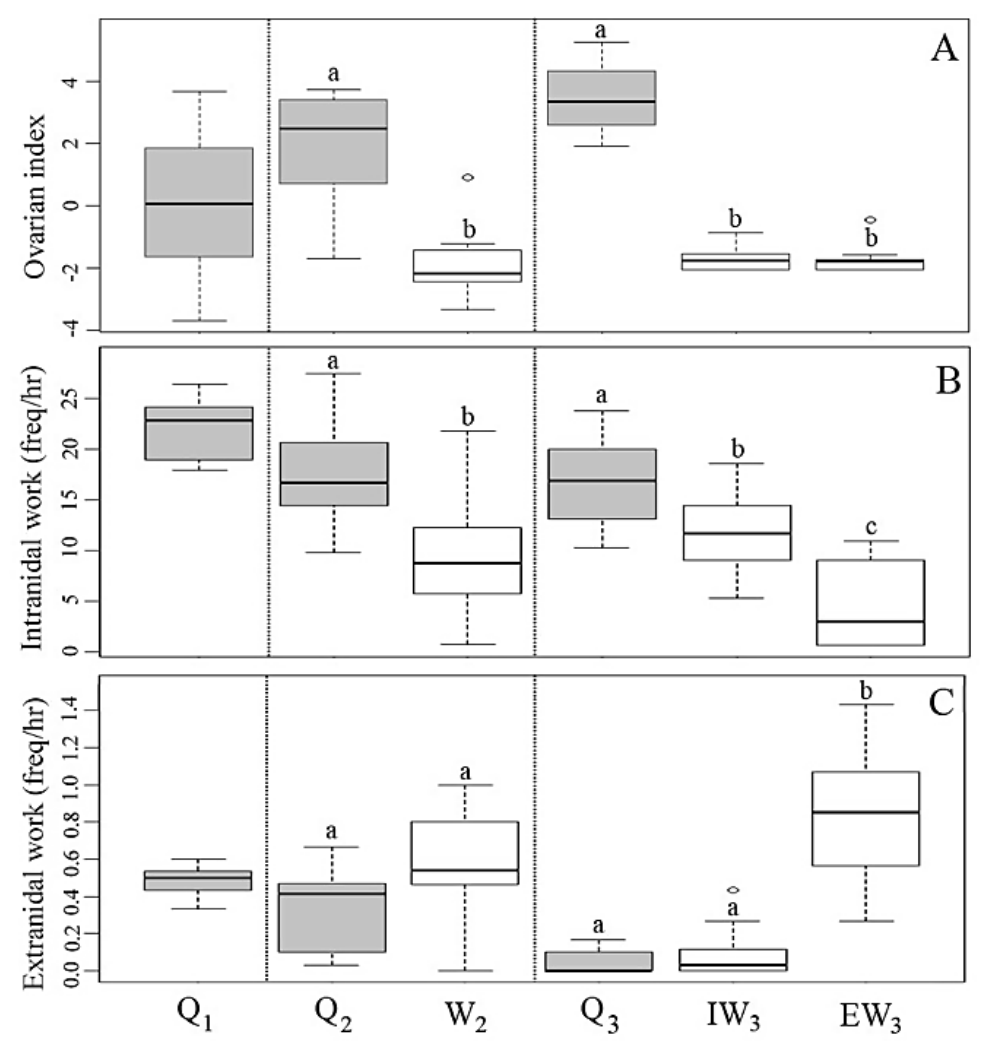

Figure 5. Comparisons between queens in solitary, pairs, and triplets and between queens and workers in pairs and triplets; shaded bars show queens and unshaded bars show workers. Letters above the bars represent comparisons between queens and workers in the same panel; bars carrying different letters are significantly different from each other. (Redrawn from A Brahma, S Mandal and $\mathrm{R}$ Gadagkar, Emergence of cooperation and division of labor in the primitively eusocial wasp Ropalidia marginata, Proc. Natl. Acad. Sci., USA. 115, pp.756-761, 2018).

the wasps sat idle in a corner and let the others do all the work. Nor was it ever the case that the non-working wasp/s interfered, much less, destroyed the work of the others. And we never had each wasp build its own separate nest; there was always only one nest in each box. This spontaneous display of cooperative behaviour is truly remarkable. We do not expect such cooperation to be displayed if we put two or three beetles, bugs or spiders together. There is clearly an innate tendency to cooperate, in this social species, even though, as we will see below, such cooperation comes at a significant cost to all but one of the wasps in each box. This observation begs the question of what is it in the genetic make-up of this and similar species that makes them behave in this manner? I hope we can find ways to answer this question in the future.

It was never the case that one of the wasps sat idle in a corner and let the others do all the work. Nor was it ever the case that the non-working wasp/s interfered, much less, destroyed the work of the others. 


\section{Reproductive Division of Labour}

In all pairs and triplets, there was a clear reproductive division of labour. One and only one individual developed her ovaries and laid eggs. A comparison of the levels of ovarian development of the singleton (which of course developed its ovaries and laid eggs), the two wasps in the pairs and the three wasps in the triplet can be seen in panel A of Figure 5. Only one wasp in each box had well-developed ovaries, while the other one or two wasps, as the case may be, had significantly poorly developed ovaries. This is the process of reproductive caste differentiation; recall that we began by asking how this came about-how do the wasps decide who will be the queen and who will be a worker? Before we answer that let us marvel at another phenomenon.

\section{Non-Reproductive Division of Labour}

In the triplets, reproductive caste differentiation resulted in one wasp with well-developed ovaries (queen) and two wasps with poorly developed ovaries (workers). We observe that the two workers display a non-reproductive division of labour between themselves, with one specializing in working at the nest (intranidal worker) and the other specializing in working away from the nest (extranidal worker). This can be seen from panels $\mathrm{B}$ and $\mathrm{C}$ in Figure 5. Note that in this and in Figure 6, we use the following convention to label the wasps. Queens are labelled Q1, Q2

The queen still does more intranidal work compared to her two workers, but the intranidal worker does significantly more intranidal work than the extranidal worker. and Q3, in the boxes with one, two and three wasps respectively. Workers in boxes with two wasps are labelled as W2. The two workers in the triplets are labelled as IW3 and EW3, the IW and EW referring to intranidal worker and extranidal worker respectively, and the subscript 3 , referring to the fact that they are in the triplets. The queen still does more intranidal work compared to her two workers, but the intranidal worker does significantly more intranidal work than the extranidal worker. And the extranidal worker does significantly more extranidal work than either the queen or the intranidal worker. We expect that the queen will stop doing intranidal work altogether with the addition of more wasps 


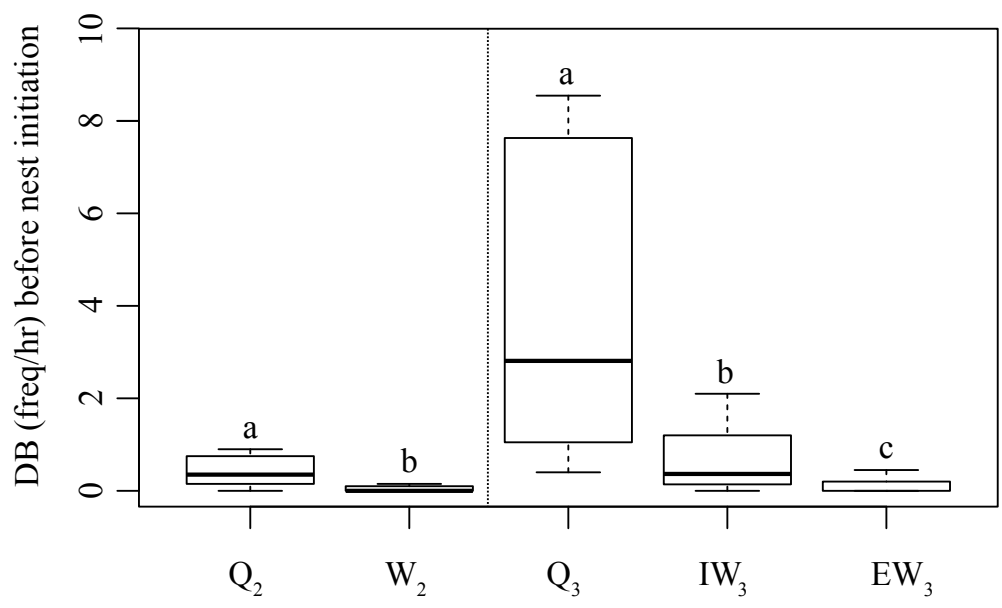

to the colony, an experiment that we are planning for the future. Just as in the case of the differentiation into queens and workers, we can also inquire into the proximate mechanism of such nonreproductive division of labour-how do the two workers decide who will specialize in the less risky intranidal work and who will specialize in the riskier extranidal work?

\section{Why Do Wasps Fight?}

The many interesting behaviours that we see in the wasp colony can be roughly classified into three kinds - those that benefit the nest and its brood, for example, building the nest, cleaning it or feeding the larvae; those that involve cooperation between the wasps, such as exchange of food or building material, mutual grooming, etc., and finally those that appear, at least at first sight, to be an expression of conflict between the wasps and can be thought of as acts of aggression, although of varying intensities. Wasps may attack, nibble, peck, chase, or immobilize each other. These behaviours are collectively labelled as 'dominance behaviour' while the reciprocal behaviours of being attacked, nibbled, pecked, being chased or being immobilized, are labelled as 'subordinate behaviour'. The expression of such dominancesubordinate behaviour is a distinct feature of primitively eusocial insects and was first discovered by the Italian zoologist, Leo
Figure 6. Comparison of rates of dominance behaviour between queens and workers in pairs and triplets. Queens show more dominance behaviour than workers in pairs (left panel), and queens show the highest dominance behaviour and extranidal workers show the least, with intranidal workers being in between (right panel). Bars carrying different letters are significantly different from each other; statistical comparisons are only within each panel. (Redrawn from A Brahma, S Mandal and R Gadagkar, Emergence of cooperation and division of labor in the primitively eusocial wasp Ropalidia marginata, Proc. Natl. Acad. Sci., USA, 115, pp.756-761, 2018.) 
Pardi. In our study, we measure the number of times per hour at which the wasps show each of the dominance or subordinate behaviours. Adding up the frequencies of the individual types of dominance behaviours such as attack, peck, etc., we get a composite number that we refer to as the frequency per hour of dominance behaviour (DB). But why do the wasps show dominance behaviour-why do they fight?

\section{The Mechanism of Division of Labour}

The wasps fight in order to decide who would be the queen, and who would be a worker. In the pairs, the winner becomes the queen and the loser becomes the worker. Here we define the winner as the one who shows more dominance behaviour and the loser as the one who shows less dominance behaviour. The two workers in the triplets also decide who would be the intranidal worker and who would be the extranidal worker by fighting.
Remarkable as it may seem, the answers to the two questions we have been seeking are intertwined. The wasps fight in order to decide who would be the queen, and who would be a worker. Put in another way, they decide who would be the queen and who would be a worker by fighting. In the pairs, the winner becomes the queen and the loser becomes the worker. Here we define a winner as the one who shows more dominance behaviour and the loser as the one who shows less dominance behaviour (Figure 6 , left panels). The two workers in the triplets also decide who would be the intranidal worker and who would be the extranidal worker by fighting. While queens show the highest levels of dominance behaviour, extranidal workers show the lowest levels of dominance behaviour, and the intranidal workers are in between (Figure 6, right panel). Notice that these different rates of dominance behaviour have been obtained from the video recordings before nest initiation. This simple experiment has answered the question raised in the title, namely, how do wasps decide who would be the queen? Indeed, it has also answered the question of how the worker wasps decide who would be the intranidal worker and who the extranidal worker, and the question of why the wasps fight. More technically speaking, this experiment has shown that fighting or dominance behaviour is the proximate mechanism of both reproductive and non-reproductive division of labour. Once the division of labour has been established, dominance behaviour reduces and what remains begins to serve an altogether different function, as we will see in the next article. 


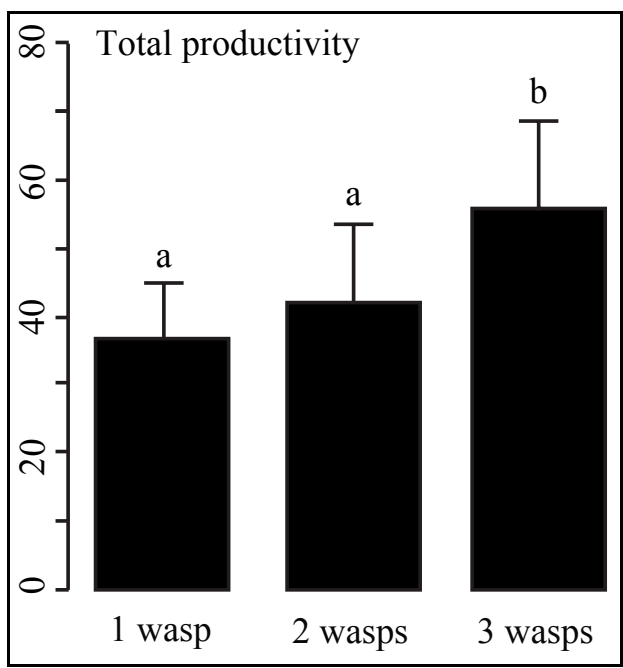

The Evolutionary Advantage of Division of Labour

Not only has this simple experiment answered the question of how the wasps decide who would be the queen and who would be the workers, and the question of who would be the intranidal workers and who the extranidal workers, but the very same experiment also answers the ultimate (evolutionary) question of the advantage of division of labour. This was possible because, although it was not strictly necessary for the original question, Anindita and Souvik kept careful records of the growth of the nests and their contents, until the nests were abandoned or were successful in producing at least one adult offspring. We define productivities of the nests by adding up the numbers of eggs, larvae of different stages and pupae contained in them on the day of eclosion of the first adult offspring. Since eggs, different stages of larvae and pupae correspond to very different amounts of work that have gone into their production, we cannot consider them as equal in computing total productivity. As a first approximation, we multiplied different developmental stages of the brood with increasing numbers (weights), as they became more advanced in age (and, by implication, needed more effort to produce). Thus, we computed total productivity as the number of eggs multiplied by 1.0, plus the number of larvae multiplied by $2.0,3.0$, and 4.0 depending
Figure 7. Comparison of boxes with 1,2 and 3 wasps in their total productivity. Total productivity is measured as the sum of the numbers of eggs, larvae and pupae (including those eclosed) on the day of the eclosion of the first adult wasp from the nest. Bars carrying different letters are significantly different from each other by the Mann-Whitney test, $\mathrm{P}<0.01)$. There is no significant difference in total productivity between one and two wasps but total productivity in three-wasp nest is significantly greater than that in one- and two-wasp nests, demonstrating the advantage of non-reproductive division of labour. (Redrawn from A Bhadra, S Mandal and R Gadagkar, Emergence of cooperation and division of labor in the primitively eusocial wasp Ropalidia marginata, Proc. Natl. Acad. Sci., USA, 115, pp.756-761, 2018.) 
Comparing across singletons, pairs and triplets, we find clear evidence of the advantage of non-reproductive division of labour-triplets had significantly higher levels of total productivity as compared to singletons and pairs. on the stage of development, plus the number of pupae multiplied by 5.0. Comparing across singletons, pairs and triplets, we find clear evidence of the advantage of non-reproductive division of labour - triplets had significantly higher levels of total productivity as compared to singletons and pairs (Figure 7). Triplets did not just have significantly higher productivity than singletons and pairs on the last day of the experiment. They showed a consistent trend of being more productive than singletons and pairs throughout the experiment (Figure 8). Thus, two wasps are adequate to cooperatively build a nest and achieve reproductive division of labour, but a significant benefit in terms of increase in productivity needs at least three wasps, and the associated division of non-reproductive labour-it needs one wasp to specialize in intranidal work and another in extranidal work. Remarkably, the division of non-reproductive labour and increase in productivity is achieved as soon as there are three wasps, the minimum number required for non-reproductive division of labour. In other words, three wasps are both necessary and sufficient for the emergence of non-reproductive division of labour and the associated increase in productivity. This is an important result because, while there are many theoretical arguments and models which postulate the advantages of non-reproductive division of labour, this little experiment provides a rare and clear empirical evidence in support of these theoretical claims.

\section{Reflections}

The very simple experiment described here required no sophisticated equipment, nor any facilities that are hard to put together. Even the video recording required no more than a simple and inexpensive webcam costing about Rs. 4000. Not having to spend time and effort for obtaining funds and setting up laboratory facilities, we can focus more carefully and completely on the design of the experiment itself. Here I want to highlight two different themes. 


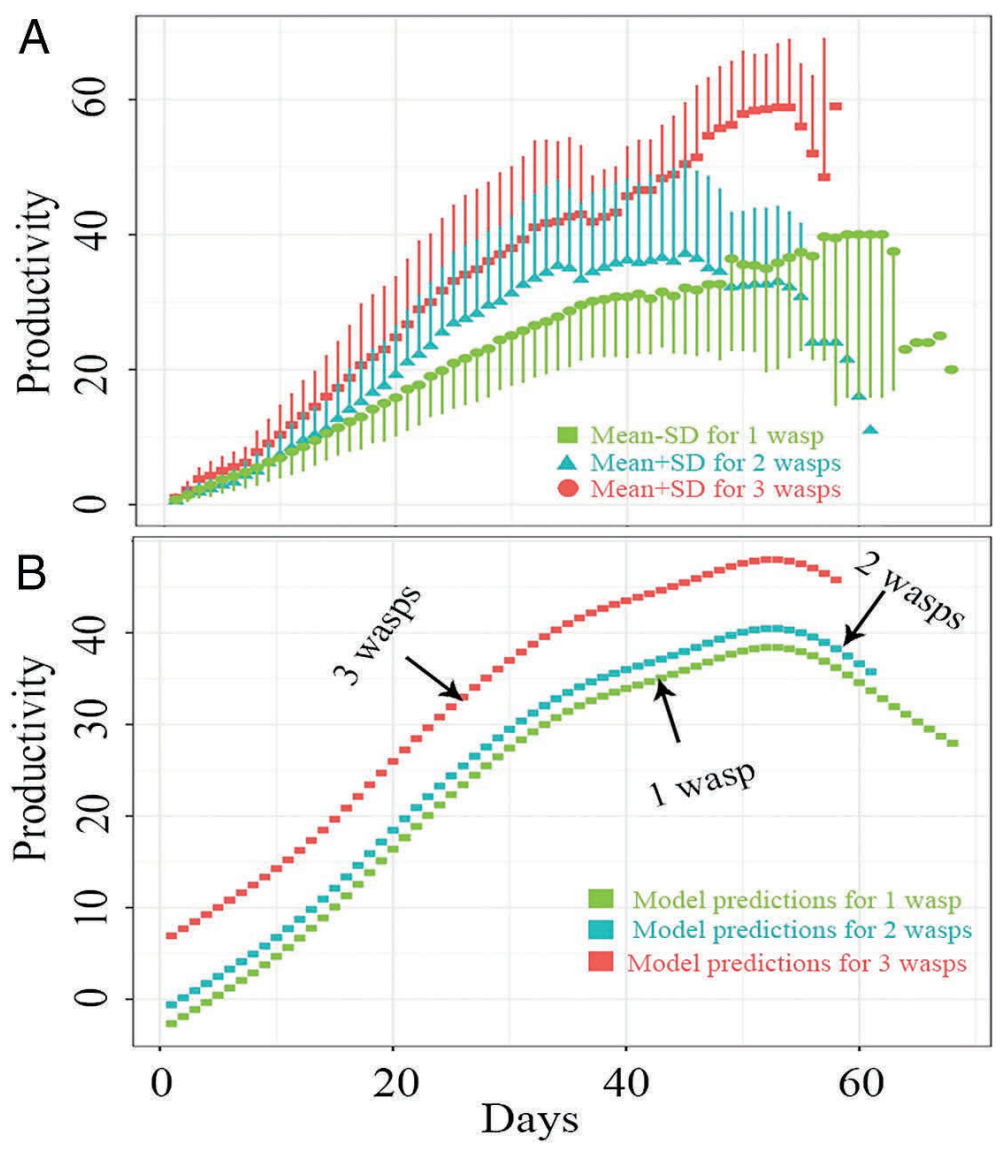

Context

We must exercise caution in asking questions and drawing conclusions about the function of animal behaviour. Animal behaviour often tends to be context-dependent - the same behaviour may have different functions in different contexts. Hence, we should be aware of the context before deciding the possible function of different behaviours. In this experiment, we asked questions such as how do the wasps decide who would be their queen, and why do they fight? We studied the wasps in the context of their building new nests and concluded that the wasps decide who would be the queen and who would be the worker, by fighting and that the function of fighting is, therefore, to settle the important question of who would be the queen. This conclusion is only in
Figure 8. Rate of change of productivity with time in singletons, pairs and triplets. Mean plus or minus standard deviation of daily productivity (upper panel) and predictions of the mean daily productivity, using a generalized additive mixed model (lower panel) See [5] for details. (Redrawn from A Brahma, S Mandal and $\mathrm{R}$ Gadagkar, Emergence of cooperation and division of labor in the primitively eusocial wasp Ropalidia marginata, Proc. Natl. Acad. Sci., USA, 115, pp.756-761, 2018.) 
Wasps also have to decide which one of them would be the queen in another context, namely, when the queen of a mature colony dies and has to be replaced by one of the workers. Although we see that the wasps show the same kinds of dominance-subordinate behaviours in mature colonies, we should not conclude that queen succession in mature colonies is also decided by fighting, nor that the function of fighting in mature colonies is to decide their next queen. the context studied here, namely, new nest foundation. Wasps also have to decide which one of them would be the queen in another context, namely, when the queen of a mature colony dies and has to be replaced by one of the workers. Although we see that the wasps show the same kinds of dominance-subordinate behaviours in mature colonies, we should not conclude that queen succession in mature colonies is also decided by fighting, nor that the function of fighting in mature colonies is to decide their next queen. We should not jump to such a conclusion even if this is indeed the correct answer in other species that have been studied so far. We will see in the next article that dominance-subordinate behaviour in mature colonies of $R$. marginata indeed has a different function and that we must, therefore, ask how wasps decide who would be the queen, all over again in that context. Recall "caution in coming to conclusions" in the definition of ethology by Peter and Jean Medawar, that has been serving us as a guiding beacon, throughout this series [6].

\section{Natural versus Artificial}

An important feature of the experiment described here is that it was conducted in rather artificial conditions. We believe that by putting wasps in acrylic boxes and giving them food and building material, we have simulated the conditions for new nest foundation in nature. Nevertheless, these conditions are very artificial. The wasps are confined to a small box and have no choice of what to eat and what to build their nest with. More importantly, they have neither the choice of being alone or being in groups nor of the choice of group size. Even more importantly, they have no choice in the matter of who their partners and cooperating companions would be. We randomly assigned wasps to live as singletons, pairs or triplets. Besides, the wasps were virgin, which may not usually be the case when they set out to build new nests in nature. Is so much artificiality permissible? Is the experiment doomed from the start? There are many discussions and misunderstandings in the literature about how natural the experimental conditions should be and how much artificiality we can afford. 
The answer really depends on the questions we are asking and the tolerance of the experimental animal to the artificiality being imposed.

Consider the virginity of the wasps we used. We know from extensive previous research that in $R$. marginata, mating is not essential for a wasp, to develop her ovaries, lay viable haploid eggs, and become the sole egg layer of a colony, even superseding other mated wasps. When a virgin and a mated wasp are kept together in small boxes, we find they each have equal probabilities of becoming the queen and workers-presumably, their fighting abilities are unaffected by their virgin or mated status. Hence, even though wasps building new nests in nature might be mated rather than virgin, we decided that it is perfectly reasonable to use virgin wasps for the experiment described here. To use only mated wasps would have made our experiment much more difficult, and would certainly have introduced other, even more undesirable artificialities.

One of the reasons we artificially confined randomly chosen wasps in small enclosures immediately after their eclosion is that we wanted to observe all their behavioural interactions from the beginning. This would not have been possible in nature. In spite of obtaining such clear-cut results about how the wasps decided who would be the queen and who would be the worker, we were constantly in search of ways of confirming these results under more natural conditions. More recently, we have succeeded in doing so. The problem in studying new nest foundations in nature, apart from the difficulty of finding them, is that we do not know where wasps seen at new nests came from. We do see wasps leaving mature colonies, but we do not know where they go to build new nests. In our closed laboratory cages, we seldom observe new nest foundation, presumably because there is insufficient space for the co-existence of multiple nests. We have now overcome these problems in a simple way. I constructed large walk-in cages measuring $1.75 \mathrm{~m} \times 1.75 \mathrm{~m} \times 1.95 \mathrm{~m}$ inside which I could place both the nest and my student Anindita (Figure 9)! As I had hoped, we immediately began to observe new nest initiations by wasps
There are many discussions and misunderstandings in the literature about how natural the experimental conditions should be and how much artificiality we can afford. The answer really depends on the questions we are asking and the tolerance of the experimental animal to the artificiality being imposed. 
Figure 9. Anindita Brahma making observations inside the walk-in cage. [Photo credit: Souvik Mandal].

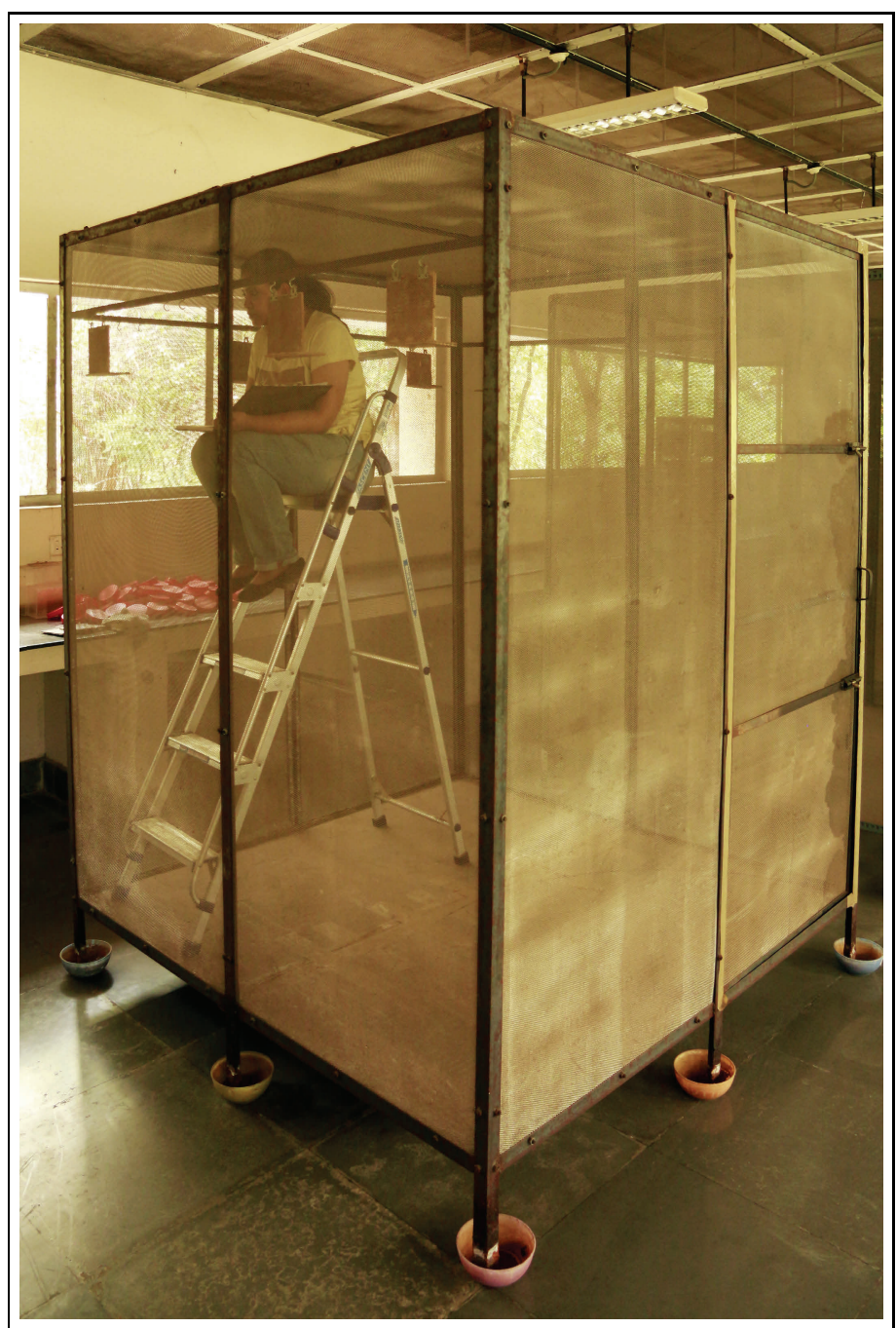

leaving their parent nests. Anindita and Souvik collected nine large nests from nature and transplanted each one of them into such walk-in cages and observed a total of 29 new nest initiations [7]. They were able to record a detailed timeline of events in each cage. Thus, we were able, for the first time, to study the process of wasps leaving their nest of birth and found new nests.

Of the 29 new nests initiated, nine were single foundress nests and 20 were multiple foundress nests. Now, in this more natural situation, we were able to understand who left the parent nest 


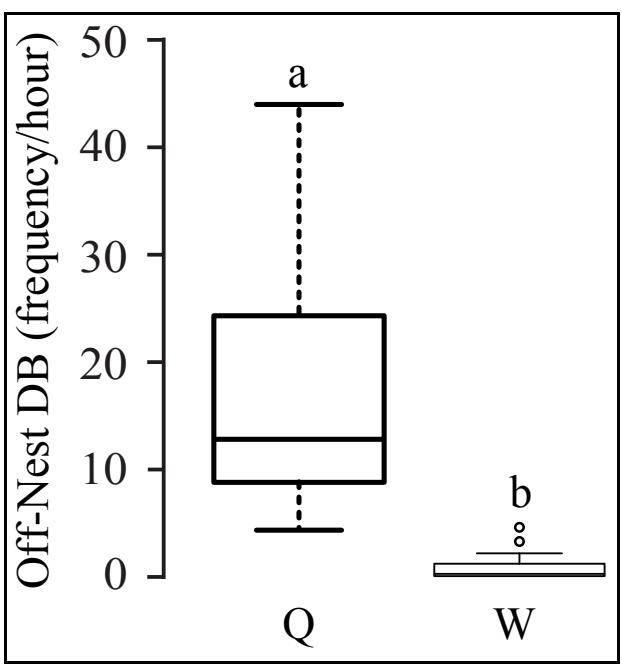

and why, who went with whom to the new nest and how they sorted themselves into queens and workers in the new nest. The most interesting result, from the point of view of the more 'artificial' experiment described in this article, is that small groups of wasps aggregated outside their parent nests and indulged in dominance-subordinate interactions even before they converged on a new place to build a new nest. The most dominant wasps in these off-nest aggregations, i.e., those showing the highest rates of dominance behaviour, became queens in the new nest, and those showing significantly lower rates of dominance behaviour became workers, thus confirming the result of the present experiment that dominance-subordinate behaviours are used to decide who would be the queen (Figure 10). It also confirmed our suspicion that the wasps might have interacted with each other and made their decisions before arriving at the new nesting site. The more natural, but more difficult, walk-in cage experiment with small sample sizes validated our more artificial, easier experiment with larger sample sizes. We were, therefore, justified in doing the artificial experiment after all. And we kept thinking of how to make it more natural, and we did. One might argue that the walk-in cage experiment is also not entirely natural-it will do for the time being, but we will keep working for ever on better experiments.
Figure 10. Dominance behaviour in off-nest aggregations in the walk-in cages, prior to new nest initiation. Future queens show significantly higher rates of aggression compared to future workers, in the off-nest aggregations in the walk-in cages, as indicated by different letters above the bars (Redrawn with permission from A Brahma, S Mandal and R Gadagkar, To leave or to stay: direct fitness through natural nest foundation in a primitively eusocial wasp, Insectes Sociaux, 66, pp.335-342, 2019). 


\section{The Future}

The 'artificial' experiment in acrylic boxes gave us the answers to our question. But they also motivated us to verify the answers in more natural settings. The walk-in cages did just that. Now they motivate us to ask the next set of questions using the 'artificial' acrylic boxes. If two wasps are adequate to produce cooperation and reproductive division of labour and three wasps are adequate to also produce non-reproductive division of labour and increase in productivity, what would be the effect of the 4th, 5th or 6th wasp on the nest? In nature, newly initiated nests can contain up to 22 wasps. We now propose to make careful studies of the behaviour of 4, 5, 6 and more wasps and hope to literally witness the emergence of social complexity, under our very eyes. The best experiments are those that while answering one question, raise one or more new ones.

\section{Acknowledgements}

I thank Anindita Brahma and Souvik Mandal for helpful comments on a draft of this article.

\section{Suggested Reading}

[1] R Gadagkar, How to design experiments in animal behaviour-6, Why are male wasps lazy? Resonance - journal of science education, Vol.24, No.9, pp.9951014, 2019.

[2] R Gadagkar, The Social Biology of Ropalidia marginata: Toward Understanding the Evolution of Eusociality, Harvard University Press, Cambridge, Massachusetts, USA, 2001.

[3] R Gadagkar, Survival Strategies: Cooperation and Conflict in Animal Societies, Harvard University Press, Cambridge, Massachusetts, USA and Universities Press, Hyderabad, India, 1997.

[4] R Gadagkar, C Vinutha, A Shanubhogue and A P Gore. Pre-imaginal biasing of caste in a primitively eusocial insect, Proceedings of the Royal Society of London B, 233, pp.175-189, 1988.

[5] A Brahma, S Mandal and R Gadagkar, Emergence of cooperation and division of labor in the primitively eusocial wasp Ropalidia marginata, Proc. Natl. Acad. Sci., USA, 115, pp.756-761, 2018. 
[6] R Gadagkar, How to design experiments in animal behaviour-1, How wasps find their nests, Resonance - journal of science education, Vol.23, No.08, pp.871-884, 2018.

[7] A Brahma, S Mandal and R Gadagkar, To leave or to stay: direct fitness through natural nest foundation in a primitively eusocial wasp, Insectes Sociaux, 66, pp.335-342, 2019. https://doi.org/10.1007/s00040-019-00702-2.

Address for Correspondence Raghavendra Gadagkar Centre for Ecological Sciences Indian Institute of Science Bangalore 560 012, India. Email: ragh@iisc.ac.in 